\title{
Neural Correlates of Impulsivity in Healthy Males and Females with Family Histories of Alcoholism
}

\author{
Elise E DeVito*,', Shashwath A Meda ${ }^{2}$, Rachel Jiantonio², Marc N Potenza ${ }^{1,3,4}$, John H Krystal, ${ }^{1,5}$ and \\ Godfrey D Pearlson ${ }^{1,2,3}$ \\ 'Department of Psychiatry, Yale University School of Medicine, New Haven, CT, USA; ${ }^{2}$ Olin Neuropsychiatry Research Center, Institute of \\ Living at Hartford Hospital, Hartford, CT, USA; ${ }^{3}$ Department of Neurobiology, Yale University School of Medicine, New Haven, CT, USA; \\ ${ }^{4}$ Child Study Center, Yale University School of Medicine, New Haven, CT, USA; ${ }^{5}$ Clinical Neuroscience Division, VA National Center for PTSD, VA \\ Connecticut Healthcare System, West Haven, CT, USA
}

Individuals family-history positive (FHP) for alcoholism have increased risk for the disorder, which may be mediated by intermediate behavioral traits such as impulsivity. Given the sex differences in the risk for and clinical presentation of addictive disorders, risk for addiction may be differentially mediated by impulsivity within FHP males and females. FHP $(N=28)$ and family-history negative $(\mathrm{FHN}, \mathrm{N}=3 \mathrm{I}$ ) healthy, non-substance-abusing adults completed an $\mathrm{PMRI}$ Go/No-Go task and were assessed on impulsivity and alcohol use. Effects of family history and sex were investigated as were associations between neural correlates of impulse control and out-ofscanner measures of impulsivity and alcohol use. FHP individuals showed greater activation in the left anterior insula and inferior frontal gyrus during successful inhibitions, an effect that was driven primarily by FHP males. Higher self-reported impulsivity and behavioral discounting impulsivity, but not alcohol use measures, were associated with greater BOLD signal in the region that differentiated the FHP and FHN groups. Impulsivity factors were associated with alcohol use measures across the FHP and FHN groups. These findings are consistent with increased risk for addiction among FHP individuals being conferred through disrupted function within neural systems important for impulse control.

Neuropsychopharmacology (2013) 38, I854-1863; doi:I0.1038/npp.20।3.92; published online I May 20।3

Keywords: impulsivity; family history of alcoholism; insula; inferior frontal gyrus (IFG); addiction; sex differences.

\section{INTRODUCTION}

Alcohol use disorders (AUDs) are prevalent and associated with detrimental health and societal outcomes ( $\mathrm{Li}, 2008)$. Individuals with a family history of alcoholism (familyhistory positive (FHP)) have increased risk for alcoholism driven by genetic and environmental factors (Lieb et al, 2002; Slutske et al, 2002). Healthy FHPs model addiction vulnerability without confounding effects of excess alcohol on cognition and brain function.

\footnotetext{
*Correspondence: Dr EE DeVito, Department of Psychiatry, Yale University School of Medicine, Suite 70 I, I Church Street, New Haven, CT 065 I0, USA, Tel: +I 203737 4882, Fax: +I 203737 359l, E-mail: elise.devito@yale.edu

Components of this data were previously presented at conferences and published in abstract form (DeVito, EE; Meda, SA; Potenza, MN; Pearlson, GD (2010): Response Inhibition in Healthy Individuals with a Family History of Alcoholism. Biol Psych 67 (9) Supplement S: I70 SI70 S.; DeVito, EE; Meda, SA; Potenza, MN; Krystal, J; Pearlson, GD (2010): The Effects Of Memantine On The Neural Correlates Of Response Inhibition In Healthy Individuals With A Family History Of Alcoholism ACER 34(6) Supplement 2: 24A-24A.)

Received 13 August 20 I2; revised 8 February 20I3; accepted I I March 2013; accepted article preview online 12 April 2013
}

Increased AUD risk among FHPs may be conferred via heritable impulsive tendencies (Dick et al, 2010; Tessner and Hill, 2010). Impulsivity is a multi-factorial construct, where distinct sub-components appear to have differential neural bases and relationships with addiction (de Wit, 2009; Lejuez et al, 2010; Rogers et al, 2010). Aspects of impulsivity may both predispose to and be exacerbated by addictive behaviors. Impulsivity predicts development of AUDs; is higher in FHPs with more alcohol-dependent relatives (ie higher family-history load); and partially mediates the relationship between FHP and alcohol and substance use disorder (for review, see de Wit, 2009; Dick et al, 2010; Lejuez et al, 2010; Verdejo-Garcia et al, 2008). Other less well-studied impulsivity-related constructs (eg, sensationseeking, risk-taking, compulsivity, behavioural activation/ inhibition, reward/punishment sensitivity) have been related to addiction vulnerability, and addictions therefore warrant consideration (eg, Meda et al, 2009).

Sex differences are important to consider as they are observable for rates, clinical presentation, and health consequences of addiction (Becker and $\mathrm{Hu}, 2008$ ) and for alcoholism-vulnerability factors, including impulsivity components (Cross et al, 2011). Although males and females demonstrate heritable alcoholism risk, they may differ on 
risk-transmission mechanisms (McGue et al, 2001; Pickens et al, 1991; Prescott et al, 1999).

Neuroimaging Go/No-Go studies have identified functional abnormalities consistent with increases in aspects of impulsivity (eg poor response inhibition) in FHPs. Healthy FHP youths display greater activity than individuals without a family history of AUDs (family-history negative (FHN)) in the middle frontal gyrus during Go/No-Go successful inhibitions (Schweinsburg et al, 2004). FHPs with and without AUDs and individuals without personal or family histories of AUDs were compared during fMRI Go/No-Go performance (Heitzeg et al, 2010). FHPs deactivated ventral caudate less than FHNs during successful inhibitions and deactivation correlated with fewer externalizing behaviors (Heitzeg et al, 2010). FHPs with AUDs deactivated orbitofrontal and medial prefrontal regions less than those without AUDs (with or without a family history), and activation correlated with alcohol use (Heitzeg et al, 2010).

In this study, we compare healthy FHP and FHN males and females on fMRI Go/No-Go, self-reported alcohol use, and extensive out-of-scanner impulsivity-related measures. Go/No-Go is a well-established measure of prepotent response inhibition, yet likely also engages cognitive processes, such as sustained attention and set maintenance (Stevens et al, 2007). The neural circuitry of this task has been well-characterized in healthy individuals (Stevens $\mathrm{et} \mathrm{al}$, 2007). Pre-specified regions-of-interest (bilateral junction of the anterior insula and inferior frontal gyrus (insula/IFG), thalamus, and left ventral caudate) were chosen for relevance to impulsivity and addiction and Go/No-Go task activation (Heitzeg et al, 2010; Stevens et al, 2007). The insula/IFG is integral to response inhibition (Garavan et al, 1999; Rubia et al, 2001; Stevens et al, 2007). The insula has been proposed to be relevant to addiction, given its role in interoception and integration of information from regions important for cognitive-control and affective processes (Naqvi and Bechara, 2010; Paulus, 2007). The thalamus receives input from the striatum through direct and indirect pathways, which respectively serve to disinhibit and inhibit thalamic output to the cortex, which, in turn, provides positive feedback to the striatum. Thalamic nuclei actively influence dynamics of information processing and transfer and may modify cortical activity (Haber and McFarland, 2001). The ventral striatum has long been a candidate structure for addiction vulnerability given its central role in salience-processing, reward-learning, acute response to illicit drugs and alcohol, and demonstrated abnormalities in addicted populations (Koob and Volkow, 2010). The left ventral striatum also contributes to Go/No-Go performance in a manner sensitive to FHP (Heitzeg et al, 2010), consistent with findings suggesting a role for the left ventral striatum in hyperactive behaviors (Martinaud et al, 2009).

To account for impulsivity's multi-factorial nature and the relevance of related constructs to addiction vulnerability, extensive out-of-scanner measures assessing impulsivity and related constructs (reward and punishment sensitivity, attention, compulsivity, risk-taking, sensationseeking) were consolidated into five factors, based on a previously published factor analysis (Meda et al, 2009). Measures of age of drinking onset and past-month alcohol consumption were explored in relation to regional FHPrelated differences in BOLD signal and impulsivity-related factors. Age of drinking onset has been associated with likelihood of later alcohol dependence and its heritability is partially mediated by impulsivity and differs by sex (McGue et al, 2001). We hypothesized that FHPs relative to FHNs would display enhanced bilateral insula/ IFG and thalamus activation, consistent with diminished inhibitory-control efficiency, and diminished ventralstriatal deactivation during Successful Inhibitions, consistent with recent findings in FHP individuals (Heitzeg et al, 2010). We hypothesized these patterns would be more robust in FHP males than females. Regarding secondary aims, we hypothesized that insula/IFG activity would correlate with out-of-scanner impulsivity-related measures, ventral-striatal activity would correlate with both impulsivity-related and alcohol-use measures, and alcohol-use measures would be associated with impulsivityrelated measures in both the family-history groups and sexes to different degrees.

\section{PARTICIPANTS AND METHODS}

\section{Participants}

Healthy FHP and FHN adults were recruited via advertisements and word-of-mouth at the Olin Neuropsychiatry Research Center, Hartford, CT. Participants provided written informed consent in accordance with the Institutional Review Boards of Hartford Hospital and Yale University. Demographic data are in Table 1.

Criteria for FHP included a father and at least one additional first- or second-degree biological relative with current or previous alcoholism according to the Family History Assessment Module. Individuals with a maternal history of alcoholism were excluded to minimize potential confounds of fetal alcohol exposure. FHN participants reported no first- or second-degree relatives with current or previous alcoholism from a family size of at least three first-degree relatives. Exclusion criteria for FHP and FHN groups included lifetime alcohol abuse or dependence, other substance use disorders (except nicotine dependence), and DSM IV-TR Axis 1 psychiatric disorders, as assessed by the Structured Clinical Interview for DSM-IV (SCID-I/NP; First et al, 2007), urine screens indicating pregnancy or recent drug use, or ethanol-positive breathalyzer results.

A 'family-history load' metric weighted AUD relatives according to the estimated shared genetic material (eg, first-degree 0.5, second-degree 0.25 ) and summed weighted scores for all AUD relatives (Stoltenberg et al, 1998) (Table 1).

Timeline follow-back assessed the total number of standard drinks in the past 30 days (henceforth 'pastmonth drinks'). Age of onset of regular (monthly) alcohol use (henceforth 'drinking-onset age') (Table 1), nicotinedependence, and lifetime marijuana, cocaine, heroin, and amphetamine use were assessed (Supplementary Table S3). NIAAA Task Force on Recommended Alcohol Questions (October 15-16, 2003) (ie frequency of past-year drinking episodes; number of drinks on a typical drinking day; number of binge-drinking episodes) data are presented in Supplementary Table S1. 
Table I Demographics, Alcohol Use and In-Scanner Behavior

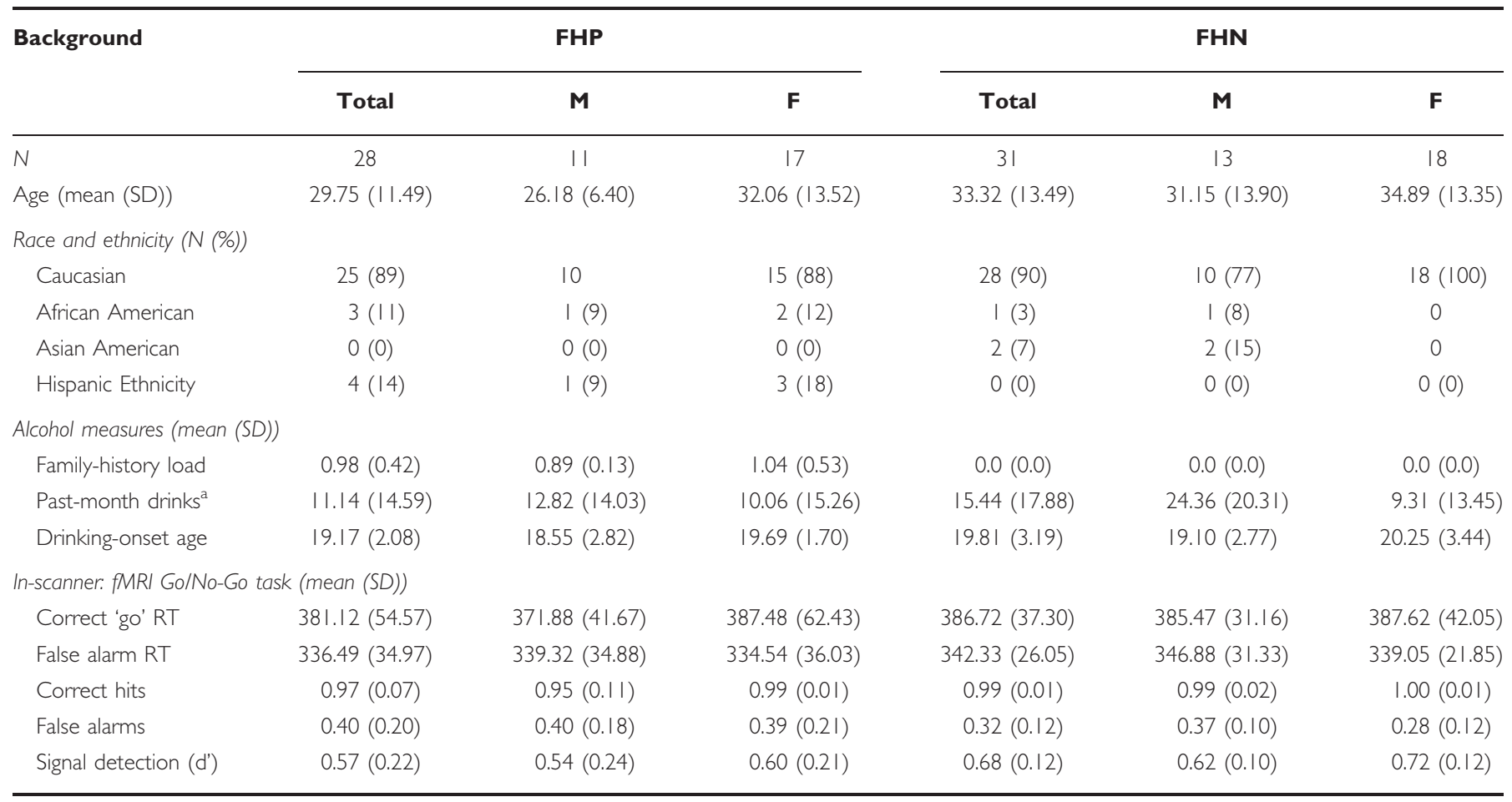

$\mathrm{FHP}=$ family history of alcoholism; FHN = no family history of alcoholism.

Data are presented as means (SD) or N (\%). Reaction times (RTs) are reported in milliseconds. Correct hits and false alarms are reported as proportion of total 'go'

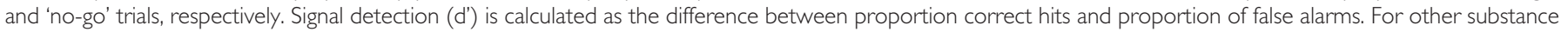
use measures, see Supplementary Table S3.

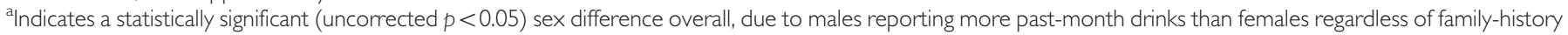
status. No other significant effects of family history, sex or family history-by-sex interactions were observed.

\section{Out-of-scanner Assessments of Impulsivity and Related Constructs}

Five impulsivity-related factors were calculated from the primary outcome measures of self-report scales and computerized risk/reward decision-making tasks; Factor 1: 'Self-reported Behavioral Activation', Factor 2: 'Self-reported Impulsivity', Factor 3: 'Self-reported Compulsivity and Reward/Punishment Sensitivity', Factor 4: 'Behavioral Temporal Discounting' and Factor 5: 'Behavioral Risk-Taking' (Table 2, Supplementary Table S4). Methods for calculating factor scores were based on a large principal component analysis conducted previously in a healthy sample (Meda et al, 2009). All participants were asked to complete five self-report scales (Barratt Impulsivity Scale Version 11 (BIS-11), Behavioral Inhibition System/ Behavioral Activation System (BIS/BAS), Padua Inventory, Zuckerman Sensation-Seeking Scale Version 5 (SSS-V), Sensitivity to Punishment and Sensitivity to Reward Questionnaire (SPSRQ)) and two computerized risk/reward decision-making tasks (Balloon Analogue Risk Task (BART), Experiential Discounting Task (EDT)). Thirteen primary outcome measures were derived from these selfreport and behavioral measures (Table 2, Supplementary Table S4). Primary outcome measures for each individual were converted to $z$-scores then multiplied by the factor weights from the study by Meda et al (2009) 'healthy-subject-only' principal component analysis to calculate five summary impulsivity factors for each individual (see Table 2, Supplementary Tables S4, S5 for complete factor analytic methods). All primary outcome measures contributed to each factor (Supplementary Table S5), but factor labeling and identification was based on outcome measures with factor weightings of $\geqslant 0.5$ (Table 2 , Supplementary Table S5). When data were missing, the factor it primarily contributed to (weighting $\geqslant 0.5$ ) was not calculated for that subject but the remaining four factors (to which the missing component measure contributed less significantly) were calculated for that subject using the group mean for the outcome measure in place of that individual's score (Supplementary Tables S4, S5).

ANOVAs with family-history status and sex were conducted for the demographic and alcohol-use measures (Table 1) and out-of-scanner impulsivity-related factors (Table 2, Supplementary Table S4). Chi-square tests compared sex distribution across groups. Where necessary, data were transformed to meet parametric assumptions or Mann-Whitney $U$ tests were utilized.

\section{Imaging Protocol}

Participants were scanned at the Olin Neuropsychiatric Research Center with a 3-Tesla head-only MRI scanner (Allegra; Siemens, Erlangen, Germany) equipped with 40$\mathrm{mT} / \mathrm{m}$ gradients and a standard quadrature head-coil. Functional imaging data were acquired using an echoplanar 
Table 2 Out-of-Scanner Impulsivity Factors

Out-of-scanner behavioral and self-report measures of impulsivity and related constructs

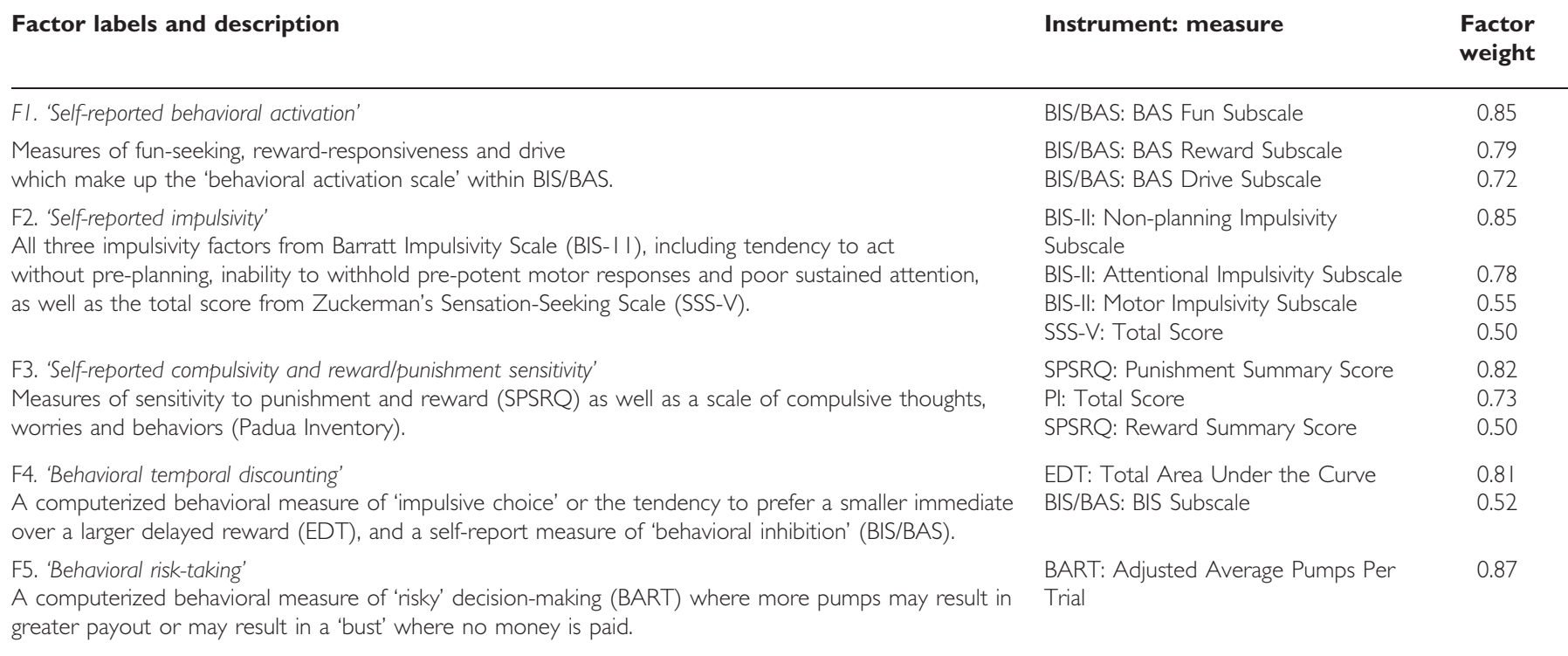

BIS/BAS: Behavioral Inhibition System/Behavioral Activation System; BIS-I I: Barratt Impulsivity Scale (Version I I); SSS-V: Zuckerman Sensation-Seeking Scale (Version 5); SPSRQ: Sensitivity to Punishment and Sensitivity to Reward Questionnaire; PI: Padua Inventory; EDT: Experiential Discounting Task; BART: Balloon Analogue Risk Task.

Out-of-scanner neuropsychological task and self-report questionnaire measures of interest are paired with the impulsivity-related factor for which they had a factor weighting of $\geqslant 0.5$, the cutoff used by Meda et al (2009) to identify the factors, although all measures contributed to some degree to all the five factors. Missing component measure data prevented calculation of F2 for one participant, F4 for six participants and F5 for one participant. For complete description of factors, component measures, and treatment of missing data, see Supplementary Materials and Meda et al (2009). Due to the contribution of EDT 'Area Under the Curve' to the calculation of F4, where a smaller area under the curve indicates more impulsive choice, smaller F4 scores also indicate more impulsivity, in contrast to the directionality of the other impulsivity-related factors. No effects of family history, sex or family history-by-sex interactions on factor scores survived corrections for multiple comparisons.

sequence with the following imaging parameters: $\mathrm{TR}=1500$ $\mathrm{ms}, \mathrm{TE}=27 \mathrm{~ms}, \mathrm{FOV}=22 \mathrm{~cm}$, flip angle $=70^{\circ}$, acquisition matrix $=64 \times 64$, voxel size $=3.44 \times 3.44$, slice thickness $=5 \mathrm{~mm}$, number of slices $=29$, ascending acquisition. Six scans performed at the beginning of each session were discarded before analysis to achieve longitudinal equilibrium. Padded cushions minimized participant movement.

\section{fMRI Task}

Following 10 out-of-scanner practice trials, participants completed two runs of an fMRI Go/No-Go task (Jamadar et al, 2012; Stevens et al, 2007). Runs each consisted of 246 trials, lasted $7 \mathrm{~min} 21 \mathrm{~s}$ and were separated by an approximately 1-min break. Participants were asked to press a button for Go (' $\mathrm{X}$ ', $85 \%$ of trials) and withhold responding to No-Go (' $K$ ', $15 \%$ of trials) stimuli. Instructors emphasized the importance of response speed and accuracy before task onset and between runs. Stimuli were presented randomly except 'K's were never presented consecutively. The inter-stimulus interval varied randomly between 650,1650 , and $2650 \mathrm{~ms}$. Stimuli were displayed on an LCD, which projected to a screen seen by participants via a mirror attached to the MRI headcoil. The screen subtended approximately a 25-degree field-of-view.

In-scanner behavioral performance was assessed with 'Correct Hit' and 'False Alarm' mean reaction time (RT) and signal detection index ( $\mathrm{d}$ ' $=$ (correct hits/total 'Go' trials $)-$ (false alarms/total 'No-Go' trials)) (Table 1).

\section{fMRI Analysis}

Functional images were reconstructed offline and analyzed with SPM5 (Wellcome Department of Imaging Neuroscience, London, UK). Runs were separately realigned then used to create one mean functional image per run, which was spatially normalized to Montreal Neurological Institute and smoothed with a $9 \mathrm{~mm}$ FWHM Gaussian kernel. Where excessive motion $(>4 \mathrm{~mm}$ ) was indicated, an artefact repair toolbox for SPM5 (ArtRepair;http://cibsr. stanford.edu/tools/ArtRepair/ArtRepair.htm) was employed (2 FHP, 2 FHN).

At first-level analysis, a canonical hemodynamic response with its temporal derivative was fitted to the onset of three events: 'Correct Hits' to 'Go' stimuli, 'False Alarms' (ie responses to 'No-Go' stimuli), and 'Successful Inhibitions' of responses to 'No-Go' stimuli. The remaining data not modelled into the events of interest comprised the implicit baseline. High frequency of 'Go' stimuli resulted in a saturated estimated response function for this trial type; therefore, 'Correct Hit' trials were not included in the contrasts. The contrasts of interest were Successful Inhibitions (successful inhibitions $>$ baseline) and False Alarms (false alarms $>$ baseline). 
For second-level analysis, a general linear model was constructed using family history (FHP, FHN) and sex (M, F) as between-subject factors for each contrast. Whole-brain analyses were first thresholded at $p_{\text {uncorrected }}<0.001, k=0$. Region-of-interest analyses examining the effects of family history, sex, and family history-by-sex were carried out in SPM5 and thresholded with a small volume correction to family-wise error (FWE) corrected $p_{\mathrm{FWE}}<0.01$. This threshold uses Bonferroni-correction to conservatively adjust the standard $p_{\mathrm{FWE}}<0.05$ to account for the use of five regionsof-interest.

The five a priori regions-of-interest were defined with spheres $(10 \mathrm{~mm}$ radii) at right insula/IFG $(33,21,0)$, left insula/IFG $(-36,18,0)$, right thalamus $(9,-12,9)$, left thalamus $(-12,-15,9)$, and left ventral striatum $(-12,24,-6)$ (Supplementary Figure S1). The location of the left ventral striatum region-of-interest was based on the reported peak FHP vs FHN group difference in an fMRI Go/No-Go study (Heitzeg et al, 2010). Other regions-ofinterest locations were based on peaks reported for this Go/No-Go fMRI task in an independent sample of healthy subjects (Stevens et al, 2007).

\section{Correlation Analyses}

Average regional brain activity was extracted from clusters showing significant $\left(p_{\mathrm{FWE}}<0.01\right)$ family-history group differences or family history-by-sex interactions (Table 3 ). Data representing the activation (in arbitrary units) of the cluster of voxels to contrasts of interest (successful inhibitions or false alarms relative to baseline) were extracted as mean cluster eigenvalues using the 'extract eigenvalues' function in SPM5. Eigenvalues were entered into SPSS 18.0 for correlations and presentation (Figures 1 and 2). Pearson's product-moment correlations evaluated associations between mean eigenvalues, impulsivityrelated factors, in-scanner signal detection (d'), drinkingonset age, and past-month drinks. The threshold for correlations was Bonferroni-corrected to $P \leqslant 0.006$ to account for the eight variables tested against each regionof-interest.

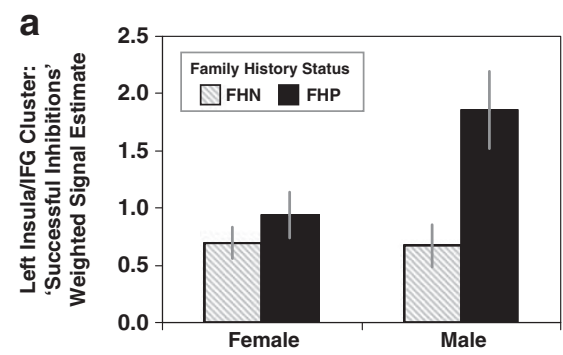

b

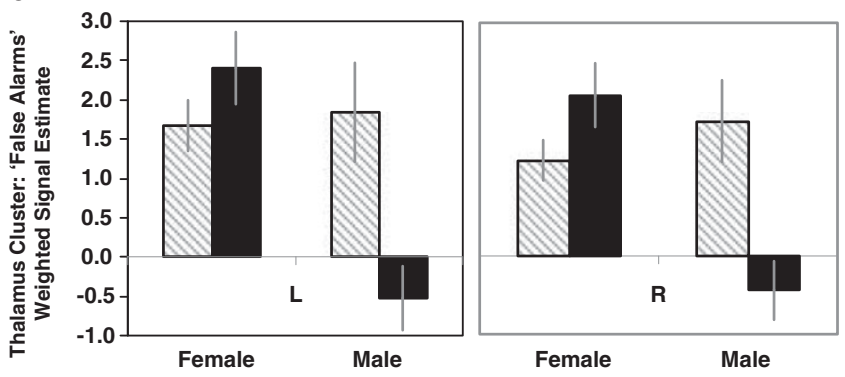

Figure I Effects of family history of alcoholism and sex on fMRI Go/NoGo. Bars represent the mean \pm SEM of the mean eigenvalues (presented in arbitrary units) extracted from each cluster, which reached significance $\left(p_{\text {FWE }}<0.0 \mathrm{I}\right.$ ) within the regions of interest (Table 3$)$. FHP $=$ positive for family history of alcoholism; $F H N=$ negative for family history of alcoholism; $L=$ left; $R=$ right; IFG = inferior frontal gyrus. (a) Hyperactivation in FHP vs FHN during successful no-go trials. The greater activity in the left insula/IFG activity during 'Successful Inhibition' trials in individuals with a family history of alcoholism (FHP) compared with those without (FHN) was primarily driven by enhanced activation in the FHP Males. (b) Family history-by-sex interaction during 'False Alarm' trials. Despite no significant main effects of family-history status or sex, the family history-by-sex interaction appeared primarily driven by diminished activity in the right and left thalamic regions in the FHP males relative to other subgroups on No-Go trials where a faulty response was made (ie 'false alarm' trials).

Table 3 fMRI Go/No-Go Group Differences

\begin{tabular}{|c|c|c|c|c|c|c|}
\hline \multirow[t]{2}{*}{ ROI } & \multirow[t]{2}{*}{ k } & \multirow[t]{2}{*}{$t$} & \multirow[t]{2}{*}{ PrWe } & \multicolumn{3}{|c|}{ Peak voxel coordinates $x, y, z$} \\
\hline & & & & $\mathbf{x}$ & $y$ & $\mathbf{z}$ \\
\hline \multicolumn{7}{|c|}{ Successful inhibition contrast } \\
\hline \multicolumn{7}{|c|}{ Main effect of family history of alcoholism (FHP > FHN) } \\
\hline $\mathrm{L}$ insula/IFG & 16 & 4.16 & 0.003 & -42 & 15 & 3 \\
\hline $\mathrm{R}$ insula/IFG & 2 & 3.53 & $0.015^{\mathrm{a}}$ & 33 & 12 & 3 \\
\hline \multicolumn{7}{|c|}{ Main effect of sex (Male > Female) } \\
\hline $\mathrm{L}$ insula/IFG & 2 & 3.52 & $0.015^{\mathrm{a}}$ & -42 & 21 & 6 \\
\hline \multicolumn{7}{|c|}{ False alarm contrast } \\
\hline \multicolumn{7}{|c|}{ Family history-by-sex interaction } \\
\hline $\mathrm{L}$ thalamus & 7 & 3.84 & 0.006 & -9 & -6 & 12 \\
\hline $\mathrm{R}$ thalamus & 29 & 4.04 & 0.004 & 15 & -15 & 15 \\
\hline
\end{tabular}

Threshold of $p_{F W E}<0.01$ Bonferroni-corrects for use of five ROls (left IFG/insula, R IFG/insula, $L$ thalamus, $R$ thalamus, ventral caudate).

$\mathrm{ROI}=$ region of interest; $k=$ cluster size in voxels; $x, y, z=M N I$ coordinates of peak voxel; $L=$ left; $R=$ right; IFG=inferior frontal gyrus; FHP = family history of alcoholism; $\mathrm{FHN}=$ no family history of alcoholism.

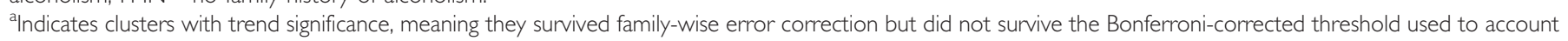
for the use of multiple ROls. 


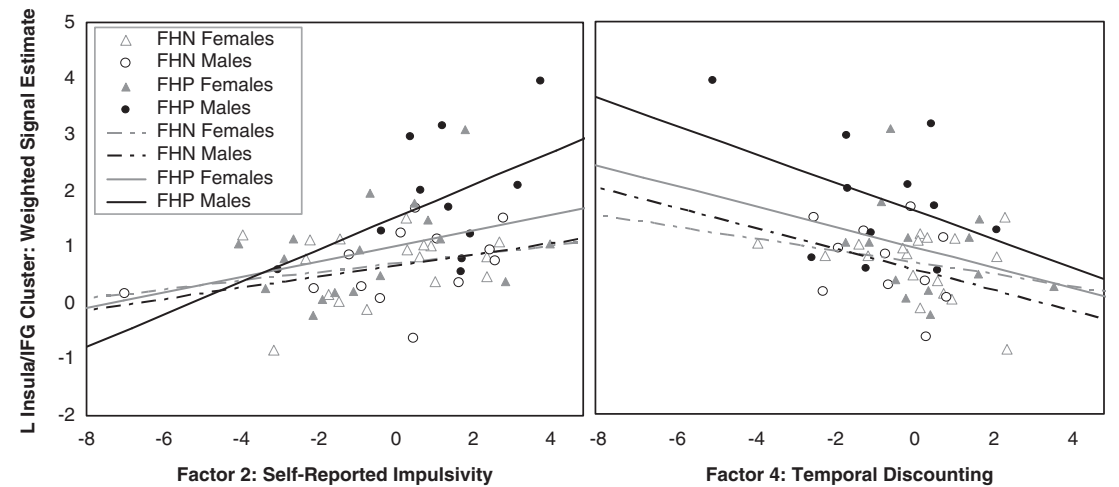

Figure 2 Left insula/IFG fMRI activity associated with greater impulsivity. Left insula/IFG activity during 'Successful Inhibitions' is associated with greater impulsivity on Factor 2 Self-Reported Impulsivity and Factor 4 Temporal Discounting. $M$ MRI activity represents the mean eigenvalue (presented in arbitrary units) extracted from the cluster within the left insula/IFG region of interest, which was significantly more activated during Successful Inhibitions in the FHP relative to FHN group (Table 3, Figure I). Larger Factor 2 scores are primarily driven by higher BIS-II scores, while smaller Factor 4 scores are primarily driven by smaller area under the curve on a delay discounting task, both of which indicate greater impulsivity. Although the association is strong in the sample overall (Factor 2: $r_{58}=0.41, p=0.001$; Factor 4: $r_{53}=-0.37, p=0.006$ ) in both cases, the association is strongest in FHP Males (solid black line, filled circles), less robust in FHP Females (solid grey line, filled triangles), and weakest in FHN males (dotted black line, empty circles) and females (dotted grey line, empty triangles).

To assess whether the relationships between impulsivityrelated and alcohol measures were influenced by family history or sex, correlations between impulsivity-related and alcohol variables were investigated within family history and sex groups. The relative strengths of the correlations were compared across subgroups using Fisher's $Z$-transformation.

\section{RESULTS}

\section{Demographics, Alcohol Use, Impulsivity-Related Factors and In-Scanner Behavior}

Family-history groups did not differ in sex distribution $\left(X^{2}=.04, p=0.84\right)$ and FHP males and females did not differ in family-history loading (Mann-Whitney $U$ Test, $p=0.50$ ). There were no significant effects of family history, sex, or family history-by-sex on age, drinking-onset age, or in-scanner reaction time for correct 'Go' or incorrect 'No-Go' (ie 'False Alarm') trials. There were no significant effects of family history or family history-by-sex on pastmonth drinks. A sex effect reflected women drinking less than men $(F=5.52, p=0.02)$ (Table 1$)$. Effects of family history and sex on out-of-scanner impulsivity-related factors did not survive corrections for multiple comparisons (Table 2, Supplementary Table S4).

\section{fMRI Results}

Effects of task were consistent with previous publications with this task (Stevens et al, 2007) with 'Successful Inhibitions' and 'False Alarm' contrasts engaging frontal, parietal, striatal, and thalamic regions (Supplementary Figure S2, Supplementary Table S2).

\section{fMRI Group Differences}

During 'Successful Inhibitions', FHPs demonstrated hyperactivity relative to FHNs in the left insula/IFG. Trends towards effects of family history in the right insula/IFG $(\mathrm{FHP}>\mathrm{FHN})$ and sex in the left insula/IFG (males $>$ females) did not survive Bonferroni's correction for multiple regions-of-interest (Table 3, Figure 1).

During 'False Alarms', despite no significant or trend main effects of family history or sex, there were significant family history-by-sex interactions in the right and left thalamus. To investigate interactions, mean eigenvalues extracted from significant clusters were compared. Bilaterally, significant family history-by-sex interactions were driven by sex differences in FHPs (Right: $t=4.18, p<0.001$; Left: $t=4.41, p<0.001$ ) but not FHNs (Right: $t=-0.92$, $p=0.36$; Left: $t=-0.26, p=0.79)$, and family-history differences within males (Right: $t=3.24, p=0.004$; Left: $t=3.03, p=0.006$ ) but not females (Right: $t=-1.69$, $p=0.101$; Left: $t=-1.32, p=0.198$ ) (Table 3, Figure 1). No other family history, sex, or family history-by-sex effects for 'Successful Inhibition' or 'False Alarm' contrasts survived corrections for multiple comparisons.

\section{Associations between fMRI, Impulsivity-Related and Alcohol Measures}

The fMRI signal eigenvalues extracted from the left insula/ IFG cluster significantly correlated with higher SelfReported Impulsivity (F2) $\left(r_{58}=0.41, p=0.001\right)$ and Temporal Discounting (F4) $\quad\left(r_{53}=-0.37, \quad p=0.006\right.$; Figure 2). The strength of these correlations between fMRI signal and impulsivity factors did not significantly differ by family history or sex. No other correlations between fMRI cluster activity and impulsivity factors or drinking measures approached statistical significance.

'Past-month drinks' was associated with Temporal Discounting (Factor 4$)\left(r_{42}=-0.42, p=0.006\right)$, and the strength of the association did not significantly differ by family history or sex.

\section{DISCUSSION}

We examined impulsivity, alcohol use measures, and fMRI Go/No-Go BOLD activation patterns in pre-selected 
regions-of-interest in healthy individuals with and without a family history of alcoholism but no personal histories of alcohol/substance use disorders. Main effects of task were consistent with previous publications (Stevens et al, 2007). While successfully inhibiting responses on 'No-Go' trials, FHPs activated the left insula/IFG region more robustly than FHNs, and this effect was more pronounced in FHP males than females. While failing to inhibit responses, family history-by-sex interactions in the bilateral thalamus reflected similar levels of thalamic activation in FHN males and females but exaggerated activation in FHP females and deactivation in FHP males. Activity in the left insula/IFG cluster correlated with aspects of impulsivity, which, in turn, were associated with alcohol use measures.

Our hypotheses that FHPs would show neuroimaging results consistent with diminished efficiency relative to FHNs in neural systems important for aspects of impulse control, and that this effect would be more pronounced in FHP males than females, was supported by the finding of increased left insula/IFG activity during successful inhibition in the FHP group, primarily driven by FHP males, and the association between greater activity in this cluster with higher out-of-scanner impulsivity scores. Hypothesized main effects of family history were not observed in other regions-of-interest.

\section{Sex Differences in Effects of Family History}

Historically, the possibility of sex differences in vulnerability mechanisms for addictions has been underemphasized; previous, similar studies have mostly not addressed this, perhaps, in part, because of sample size limitations. Family history appeared to influence the neural correlates of response inhibition more in males. As FHP females and males were well matched for family-history loading and other demographic factors, these findings may be consistent with FHP females requiring greater family-history loading to confer similar degrees of risk as males. Alternatively, if sexes differ in risk-transmission mechanisms, FHP females may demonstrate equally or more substantially disrupted function than males in neural circuitry engaged by different cognitive processes than those tapped by Go/No-Go.

Females reported fewer past-month drinks. However, the lack of associations between alcohol and fMRI measures in this sample suggests sex differences in alcohol use were unlikely to account for fMRI sex differences in FHPs.

\section{Anterior Insula/IFG and Impulsivity-related Constructs}

Neuroimaging, stimulation, and lesion studies implicate the anterior insula/IFG in response inhibition with support for a right-dominated network (eg, Aron et al, 2003; Garavan et al, 1999), which also engages the left insula/IFG to a lesser degree (eg, Rubia et al, 2001; Stevens et al, 2007). Go/No-Go activates a bilateral, yet still right-dominated, network compared with more right lateralized response inhibition tasks (ie the Stop-Signal task) (Rubia et al, 2001; Swick et al, 2008). Adolescents (Stevens et al, 2007) and elderly (Nielson et al, 2002) activate a more diffuse network during successful inhibition, including greater recruitment of the left prefrontal cortex, compared with young and middleaged adults. This may suggest that normally right-domi- nated tasks recruit greater engagement of left-sided networks when necessary to compensate for a less efficient right insula/IFG. Given the trend towards greater right insula/IFG in the FHP group, the significantly increased engagement of the left insula/IFG in the FHP relative to the FHN group may reflect recruitment of a more diffuse bilateral network in compensation for right insula/IFG inefficiency (Table 3).

The insula is implicated in impulsivity-related constructs, including, but not limited to, choice impulsivity (Tanaka et $\mathrm{al}, 2004$ ) and risk and uncertainty assessment (Clark et al, 2008). These constructs, along with response inhibition, may require integration of sensory and interoceptive cues to guide cognitive and motor responses, evaluate outcomes, and adjust behavior (Brass and Haggard, 2010). Disruption to such processes may contribute to addiction vulnerability.

\section{Insula and Addiction}

Several models of addiction emphasize a role for the insula, given its ability to influence complex motivated behaviors by integrating bodily sensations, external stimuli, and motivational states while engaging executive and motor systems. Bodily sensation information, transmitted from the spinal cord via the ventromedial thalamic nucleus, is integrated with subcortical, limbic, and cortical circuitry in the anterior insula (Craig, 2002). The anterior insula and anterior cingulate have been proposed to form a 'salience network', facilitating attention to salient internal or environmental stimuli, then engaging cognitive control processes to access working memory and attentional resources by switching between central executive and default mode networks (Menon and Uddin, 2010). As reward response is influenced by homeostatic and external contexts, the insula has been proposed to contribute to complex cognitive, affective, and behavioral phenomena in addictions (Paulus, 2007), including conscious processing of pleasurable drug effects, drug cue-related craving (Garavan et al, 2000; Gray and Critchley, 2007; Koob and Volkow, 2010), and 'as-if' pleasure and decision-making processes weighing drug-taking positive $v s$ negative consequences (Naqvi and Bechara, 2010).

\section{Family History-by-Sex Interactions in the Thalamus}

The thalamus family history-by-sex interaction was driven by sex differences in the FHP but not in the FHN group and a family-history group difference in males but not in females. The thalamus is thought to have a central role in gating information transfer along cortico-striatal-thalamocortical circuits and may actively modulate the resulting task-related cortical activation and deactivation patterns that signal a shift from non-task-related activity to task engagement (Haber and McFarland, 2001). Sex differences in associations between task-related activation (or de-activation) and response inhibition performance may indicate sex differences in task-related 'processing strategies' (Liu et al, 2012). White matter microstructural differences suggest sex differences in the thalamus's role in cortico-striatalthalamo-cortical circuit function (Menzler et al, 2011). Sex differences in thalamic activation in FHP may reflect sex differences in mechanisms of addiction vulnerability. 
Lack of Family History of Alcoholism Effects in Ventral-Striatum

Contrary to our hypothesis, our family-history groups did not differ on ventral-striatal activity. Previous findings of abnormal ventral-striatal activity in adolescent/young adult FHP or alcohol-dependent individuals were often associated with high impulsivity or alcohol use (Beck et al, 2009; Bjork et al, 2008a; Bjork et al, 2008b; Heitzeg et al, 2008; Heitzeg et al, 2010; Wrase et al, 2007). The lack of ventral-striatal findings in this sample may have been due to the FHP and FHN groups' similar impulsivity and alcohol use levels. Ventral-striatal abnormalities may only be present in FHPs with high impulsivity or excess alcohol use or may partially reflect a developmental delay observable during adolescence/young adulthood.

\section{Associations between Neural, Impulsivity-Related and Drinking Measures}

Greater left insula/IFG cluster activity was associated with higher impulsivity (self-reported impulsivity and sensation seeking (F2) and steeper delay discounting (F4)). Taken together, the trend towards greater right insula/IFG recruitment (previously associated with higher self-reported impulsivity as measured by Eysenk's Impulsivity Scale (Horn et al, 2003)) and significantly greater left insula/IFG recruitment in FHPs, and associations between left insula/ IFG activity and impulsiveness across both groups, are consistent with diminished cognitive efficiency of response inhibition processes in FHPs.

Steeper delay discounting (F4) was associated with more past-month drinking, consistent with previous research (Kollins, 2003). This impulsivity component may predispose towards alcohol use. Given the trend correlations between F4 and alcohol-onset age, an alternate explanation involving early alcohol exposure leading to increased choice impulsivity also warrants consideration (Nasrallah et al, 2009).

\section{Limitations and Future Directions}

Use of multiple out-of-scanner impulsivity constructs was a strength (Lejuez et al, 2010). However, the lack of robust effects of family history on out-of-scanner impulsivityrelated factors may reflect our strict exclusion criteria for lifetime problematic alcohol use or other psychiatric disorders, which likely excluded early-onset alcoholics. These FHPs may represent a protected subset or have vulnerability for later-onset alcoholism, which is associated with less impulsivity than early-onset alcoholism (Dom et al, 2006). Our results may not apply to earlyonset alcoholism or highly impulsive FHPs.

Findings are consistent with increased risk for addiction among FHPs conferred through diminished efficiency of neural systems important for impulse control, particularly among males. Maternal history of AUDs was an exclusion criterion to control for fetal alcohol syndrome. Genetic or environmental mechanisms of transmission of risk may differ by inheritance from same-sex or opposite-sex parents or by maternal or paternal AUDs (Lieb et al, 2002; Morgan et al, 2010). The purported risk-transmission mechanism of response inhibition inefficiency may be greater in father-toson than father-to-daughter inheritance. The current study only had the capacity to detect FHP or sex differences in neural systems sufficiently engaged by the Go/No-Go task and within regions-of-interest. Different neurocognitive mechanisms may be more sensitive to father-to-daughter risk transmission.

The analytic approach limited detection of potential family history or sex differences to a priori regions-ofinterest, chosen for relevance to addiction vulnerability, impulsivity-related constructs, and Go/No-Go task engagement. However, these regions-of-interest are not the only Go/No-Go-activated regions implicated in addiction vulnerability or impulsivity-related constructs.

The finding that FHPs displayed neural markers of diminished efficiency despite minimal indications of increased impulsivity suggests that subtle alterations of neural circuitry important for response inhibition may contribute to vulnerability to addiction, despite normal impulsivity and alcohol-use behaviors. Prospective studies investigating other at-risk populations for similar vulnerability markers could inform prevention efforts by identifying at-risk individuals before problematic drinking onset. As neural vulnerabilities may persist following prolonged abstinence, they may predict risk for relapse in treatment seekers.

\section{ACKNOWLEDGEMENTS}

This research was supported by National Institute on Alcohol Abuse and Alcoholism (NIAAA), 2P50 AA 012870, Center for Translational Neuroscience on Alcoholism and US Department of Veterans Affairs Alcohol Research Center, RO1 DA020709 from National Institute on Drug Abuse (NIDA). EED was supported by T32 AA015496 from NIAAA and K12 DA031050 from NIDA, NIAAA, Office of Research on Women's Health (ORWH) and NIH Office of the Director (OD). MNP was supported by P20 DA027844 from NIDA and RL1 AA017539 from NIAAA. The content is solely the responsibility of the authors and does not necessarily represent the official views of NIAAA, NIDA, or NIH. Funding bodies had no role in the collection, analysis, or the decision to publish these data. We thank Dr Sharna Jamadar for her helpful technical assistance.

\section{DISCLOSURE}

JHK consults for: Aisling Capital, Astellas Pharma Global Development, AstraZeneca Pharmaceuticals Biocortech, Brintnall \& Nicolini, Easton Associates, Gilead Sciences, GlaxoSmithKline, Janssen Pharmaceuticals, Lundbeck Research USA, Medivation, Merz Pharmaceuticals, MK Medical Communications, F. Hoffmann-La Roche, Sage Therapeutics, SK Holdings, Sunovion Pharmaceuticals, Takeda Industries, Teva Pharmaceutical Industries; serves on the Board of Directors for the Coalition for Translational Research in Alcohol and Substance Use Disorders, as President Elect of the American College of Neuropsychopharmacology, and as Editor of Biological Psychiatry; is on the Scientific Advisory Boards for Abbott Laboratories, Bristol-Myers Squibb, CHDI Foundation, Eisai, Eli Lilly and Forest Laboratories, Lohocla Research Corporation, Mnemosyne Pharmaceuticals, Naurex, Pfizer Pharmaceuticals, Shire Pharmaceuticals, StratNeuro Research Program at Karolinska Institute (International Advisory Board); is 
employed by Yale University School of Medicine and VA CT Healthcare System; holds patents for the following: (1) Seibyl JP, Krystal JH, Charney DS. Dopamine and noradrenergic reuptake inhibitors in treatment of schizophrenia. Patent \#:5 447 948.September 5, 1995, (2.) co-inventor with Dr Gerard Sanacora on a filed patent application by Yale University related to targeting the glutamatergic system for the treatment of neuropsychiatric disorders (PCTW O06108055A1), (3) Intranasal Administration of Ketamine to Treat Depression (patent pending). MNP reports no financial conflicts of interest with respect to the content of this manuscript yet has received financial support or compensation for the following: consulted for and advised Boehringer Ingelheim; consulted for and has financial interests in Somaxon; received research support from the National Institutes of Health, Veteran's Administration, Mohegan Sun Casino, the National Center for Responsible Gaming and its affiliated Institute for Research on Gambling Disorders, and Forest Laboratories, OrthoMcNeil, Oy-Control/Biotie, Glaxo-SmithKline, and Psyadon pharmaceuticals; participated in surveys, mailings or telephone consultations related to drug addiction, impulse-control disorders or other health topics; consulted for law offices and the federal public defender's office in issues related to impulse-control disorders; provides clinical care in the Connecticut Department of Mental Health and Addiction Services Problem Gambling Services Program; performed grant reviews for the National Institutes of Health and other agencies; guest-edited journal sections; given academic lectures in grand rounds, $\mathrm{CME}$ events and other clinical or scientific venues; and generated books or book chapters for publishers of mental health texts. The remaining authors declare no conflicts of interest.

\section{REFERENCES}

Aron AR, Fletcher PC, Bullmore ET, Sahakian BJ, Robbins TW (2003). Stop-signal inhibition disrupted by damage to right inferior frontal gyrus in humans. Nat Neurosci 6: 115-116.

Beck A, Schlagenhauf F, Wustenberg T, Hein J, Kienast T, Kahnt T et al (2009). Ventral striatal activation during reward anticipation correlates with impulsivity in alcoholics. Biol Psychiatry 66: 734-742.

Becker JB, Hu M (2008). Sex differences in drug abuse. Front Neuroendocrinol 29: 36-47.

Bjork JM, Knutson B, Hommer DW (2008a). Incentive-elicited striatal activation in adolescent children of alcoholics. Addiction 103: 1308-1319.

Bjork JM, Smith AR, Hommer DW (2008b). Striatal sensitivity to reward deliveries and omissions in substance dependent patients. Neuroimage 42: 1609-1621.

Brass M, Haggard P (2010). The hidden side of intentional action: the role of the anterior insular cortex. Brain Struct Funct 214: 603-610.

Clark L, Bechara A, Damasio H, Aitken MR, Sahakian BJ, Robbins TW (2008). Differential effects of insular and ventromedial prefrontal cortex lesions on risky decision-making. Brain 131(Pt 5): 1311-1322.

Craig AD (2002). How do you feel? Interoception: the sense of the physiological condition of the body. Nat Rev Neurosci 3(8): 655-666.

Cross CP, Copping LT, Campbell A (2011). Sex differences in impulsivity: a meta-analysis. Psychol Bull 137: 97-130. de Wit H (2009). Impulsivity as a determinant and consequence of drug use: a review of underlying processes. Addict Biol 14;: 22-31.

Dick DM, Smith G, Olausson P, Mitchell SH, Leeman RF, O’Malley SS et al (2010). Understanding the construct of impulsivity and its relationship to alcohol use disorders. Addict Biol 15: 217-226.

Dom G, Hulstijn W, Sabbe B (2006). Differences in impulsivity and sensation seeking between early- and late-onset alcoholics. Addict Behav 31: 298-308.

First MB, Spitzer RL, Gibbon M, Williams JBW (2007). Structured Clinical Interview for DSM-IV-TR Axis I Disorders-Non-patient Edition (SCID-I/P, 1/2007 revision). Biometrics Research Department, New York State Psychiatric Institute: New York, NY, USA.

Garavan H, Pankiewicz J, Bloom A, Cho JK, Sperry L, Ross TJ et al (2000). Cue-induced cocaine craving: neuroanatomical specificity for drug users and drug stimuli. Am J Psychiatry 157: 1789-1798.

Garavan H, Ross TJ, Stein EA (1999). Right hemispheric dominance of inhibitory control: an event-related functional MRI study. Proc Natl Acad Sci USA 96: 8301-8306.

Gray MA, Critchley HD (2007). Interoceptive basis to craving. Neuron 54: 183-186.

Haber S, McFarland NR (2001). The place of the thalamus in frontal cortical-basal ganglia circuits. Neuroscientist 7: 315-324.

Heitzeg MM, Nigg JT, Yau WY, Zubieta JK, Zucker RA (2008). Affective circuitry and risk for alcoholism in late adolescence: differences in frontostriatal responses between vulnerable and resilient children of alcoholic parents. Alcohol Clin Exp Res 32: 414-426.

Heitzeg MM, Nigg JT, Yau WY, Zucker RA, Zubieta JK (2010). Striatal dysfunction marks preexisting risk and medial prefrontal dysfunction is related to problem drinking in children of alcoholics. Biol Psychiatry 68: 287-295.

Horn NR, Dolan M, Elliott R, Deakin JF, Woodruff PW (2003). Response inhibition and impulsivity: an fMRI study. Neuropsychologia 41: 1959-1966.

Jamadar S, DeVito EE, Jiantonio RE, Meda SA, Stevens MC, Potenza MN et al (2012). Memantine, an NMDA receptor antagonist, differentially influences Go/No-Go performance and fMRI activity in individuals with and without a family history of alcoholism. Psychopharmacology (Berl) 222: 129-140.

Kollins SH (2003). Delay discounting is associated with substance use in college students. Addict Behav 28: 1167-1173.

Koob GF, Volkow ND (2010). Neurocircuitry of addiction. Neuropsychopharmacology 35: 217-238.

Lejuez CW, Magidson JF, Mitchell SH, Sinha R, Stevens MC, de Wit H (2010). Behavioral and biological indicators of impulsivity in the development of alcohol use, problems, and disorders. Alcohol Clin Exp Res 34: 1334-1345.

Li TK (2008). Quantifying the risk for alcohol-use and alcoholattributable health disorders: present findings and future research needs. J Gastroenterol Hepatol 23(Suppl 1): S2-S8.

Lieb R, Merikangas KR, Hofler M, Pfister H, Isensee B, Wittchen HU (2002). Parental alcohol use disorders and alcohol use and disorders in offspring: a community study. Psychol Med 32: 63-78.

Liu J, Zubieta JK, Heitzeg M (2012). Sex differences in anterior cingulate cortex activation during impulse inhibition and behavioral correlates. Psychiatry Res 201: 54-62.

Martinaud O, Perin B, Gerardin E, Proust F, Bioux S, Gars DL et al (2009). Anatomy of executive deficit following ruptured anterior communicating artery aneurysm. Eur J Neurol 16: 595-601.

McGue M, Iacono WG, Legrand LN, Elkins I (2001). Origins and consequences of age at first drink. II. Familial risk and heritability. Alcohol Clin Exp Res 25: 1166-1173.

Meda SA, Stevens MC, Potenza MN, Pittman B, Gueorguieva R, Andrews $\mathrm{MM}$ et al (2009). Investigating the behavioral and self- 
report constructs of impulsivity domains using principal component analysis. Behav Pharmacol 20: 390-399.

Menon V, Uddin LQ (2010). Saliency, switching, attention and control: a network model of insula function. Brain Struct Funct 214: 655-667.

Menzler K, Belke M, Wehrmann E, Krakow K, Lengler U, Jansen A et al (2011). Men and women are different: diffusion tensor imaging reveals sexual dimorphism in the microstructure of the thalamus, corpus callosum and cingulum. Neuroimage 54: $2557-2562$.

Morgan PT, Desai RA, Potenza MN (2010). Gender-related influences of parental alcoholism on the prevalence of psychiatric illnesses: analysis of the National Epidemiologic Survey on Alcohol and Related Conditions. Alcohol Clin Exp Res 34: 1759-1767.

Naqvi NH, Bechara A (2010). The insula and drug addiction: an interoceptive view of pleasure, urges, and decision-making. Brain Struct Funct 214: 435-450.

Nasrallah NA, Yang TW, Bernstein IL (2009). Long-term risk preference and suboptimal decision making following adolescent alcohol use. Proc Natl Acad Sci USA 106: 17600-17604.

Nielson KA, Langenecker SA, Garavan H (2002). Differences in the functional neuroanatomy of inhibitory control across the adult life span. Psychol Aging 17: 56-71.

Paulus MP (2007). Neural basis of reward and craving-a homeostatic point of view. Dialogues Clin Neurosci 9: 379-387.

Pickens RW, Svikis DS, McGue M, Lykken DT, Heston LL, Clayton PJ (1991). Heterogeneity in the inheritance of alcoholism. A study of male and female twins. Arch Gen Psychiatry 48: 19-28.

Prescott CA, Aggen SH, Kendler KS (1999). Sex differences in the sources of genetic liability to alcohol abuse and dependence in a population-based sample of U.S. twins. Alcohol Clin Exp Res 23: 1136-1144.

Rogers RD, Moeller FG, Swann AC, Clark L (2010). Recent research on impulsivity in individuals with drug use and mental health disorders: implications for alcoholism. Alcohol Clin Exp Res 34: $1319-1333$

Rubia K, Russell T, Overmeyer S, Brammer MJ, Bullmore ET, Sharma $\mathrm{T}$ et al (2001). Mapping motor inhibition: conjunctive brain activations across different versions of go/no-go and stop tasks. Neuroimage 13: 250-261.

Schweinsburg AD, Paulus MP, Barlett VC, Killeen LA, Caldwell LC, Pulido C et al (2004). An FMRI study of response inhibition in youths with a family history of alcoholism. Ann N Y Acad Sci 1021: 391-394.

Slutske WS, Heath AC, Madden PA, Bucholz KK, Statham DJ, Martin NG (2002). Personality and the genetic risk for alcohol dependence. J Abnorm Psychol 111: 124-133.

Stevens MC, Kiehl KA, Pearlson GD, Calhoun VD (2007). Functional neural networks underlying response inhibition in adolescents and adults. Behav Brain Res 181: 12-22.

Stoltenberg SF, Mudd SA, Blow FC, Hill EM (1998). Evaluating measures of family history of alcoholism: density versus dichotomy. Addiction 93: 1511-1520.

Swick D, Ashley V, Turken AU (2008). Left inferior frontal gyrus is critical for response inhibition. BMC Neurosci 9: 102.

Tanaka SC, Doya K, Okada G, Ueda K, Okamoto Y, Yamawaki S (2004). Prediction of immediate and future rewards differentially recruits cortico-basal ganglia loops. Nat Neurosci 7: 887-893.

Tessner KD, Hill SY (2010). Neural circuitry associated with risk for alcohol use disorders. Neuropsychol Rev 20: 1-20.

Verdejo-Garcia A, Lawrence AJ, Clark L (2008). Impulsivity as a vulnerability marker for substance-use disorders: review of findings from high-risk research, problem gamblers and genetic association studies. Neurosci Biobehav Rev 32: 777-810.

Wrase J, Schlagenhauf F, Kienast T, Wustenberg T, Bermpohl F, Kahnt $\mathrm{T}$ et al (2007). Dysfunction of reward processing correlates with alcohol craving in detoxified alcoholics. Neuroimage 35: 787-794.

Supplementary Information accompanies the paper on the Neuropsychopharmacology website (http://www.nature.com/npp) 